\title{
Modular flexible ureteroscopy and holmium laser lithotripsy for the treatment of renal and proximal ureteral calculi: A single-surgeon experience of $\mathbf{3 8 2}$ cases
}

\author{
ZEJUN YAN, GUOHAI XIE, HESHENG YUAN and YUE CHENG
}

\author{
Department of Urology, Ningbo First Hospital, Medical College of Ningbo University, Ningbo, Zhejiang 315010, P.R. China
}

Received September 17, 2014; Accepted July 31, 2015

DOI: $10.3892 /$ etm.2015.2703

\begin{abstract}
To determine the safety and efficacy of modular flexible ureteroscopy and holmium laser lithotripsy for the treatment of renal and proximal ureteral calculi, a retrospective chart review of a single surgeon's 3-year modular flexible ureteroscopy experience was performed. All of the patients were treated with modular flexible ureteroscopy and holmium laser lithotripsy by a single surgeon. Stone-free status was defined as no fragments or a single fragment $\leq 4 \mathrm{~mm}$ in diameter at the 3-month follow-up. The procedure number, operative time, stone-free rates, repeat usage of the multilumen catheter, and perioperative complications were documented. The present study included 215 male patients and 167 female patients, with an average age of $48.5 \pm 13.7$ years (range, $17-84$ years). The mean stone size was $11.5 \pm 4.1 \mathrm{~mm}$ (range, $4-28 \mathrm{~mm}$ ), and the mean total stone burden was $17.5 \pm 5.7 \mathrm{~mm}$ (range $15-46 \mathrm{~mm}$ ). A total of 305 patients $(79.8 \%$ ) had a stone burden $\leq 20 \mathrm{~mm}$, and 77 patients $(20.2 \%)$ had a stone burden $>20 \mathrm{~mm}$. The mean number of primary procedures was $1.3 \pm 0.2$ (range, $1-3$ ). The stone-free rate following the first and the second procedure was 73.4 and $86.9 \%$, respectively. The mean postoperative hospital stay was 3.1 \pm 1.2 days (range, $2-6$ days). The highest clearance rates were observed for proximal ureteral stones $(100 \%)$ and renal pelvic stones $(88.7 \%)$, whereas the lowest clearance rates were observed for lower calyx stones $(76.7 \%)$ and multiple calyx stones $(77.8 \%)$. The higher the initial stone burden, the lower the postoperative stone-free rate $(\leq 20$ vs. $>20 \mathrm{~mm} ; 89.8$ vs. $75.3 \%$ ). The overall complication rate was $8.1 \%$. The results of the present study suggest that modular flexible ureteroscopy with holmium laser lithotripsy may be considered the primary method for the treatment of renal and proximal ureteral calculi in select patients, due to its accept-
\end{abstract}

Correspondence to: Professor Yue Cheng, Department of Urology, Ningbo First Hospital, Medical College of Ningbo University, 59 Liuting Street, Ningbo, Zhejiang 315010, P.R. China

E-mail: 35485787@qq.com

Key words: laser lithotripsy, modular flexible ureteroscopy, renal and proximal ureteral calculi able efficacy, low morbidity, and relatively low maintenance costs.

\section{Introduction}

Since Marshall (1) initially reported the use of a flexible cystoscope to visualize a ureteric stone, there have been marked technical advancements in the flexible ureteroscope, which has revolutionized the treatment of upper urinary tract calculi. However, the conventional integrated flexible ureteroscope is fragile and costly, and the ongoing maintenance and repair costs are expensive. Afane et al (2) assessed four flexible ureteroscopes and reported that repair of flexible ureteroscopes was mandatory after an average of 6-15 uses or 3-13 h. Similarly, Landman et al (3) evaluated the durability of the ACMI DUR-8 ureteroscope and noted continued function in 25 cases prior to repair. Carey et al (4) reported 40-48 uses for four flexible ureteroscopes, including ACMI DUR-8, DUR-8 Elite, and Storz Flex-X, prior to the first repair. In addition, Canales et al (5) reported the average repair cost for flexible ureteroscopes to be 4,597 USD, which may have since risen.

Flexible ureteroscopes also require backup equipment, due to possible malfunction, which necessitates further financial commitment. All of these disadvantages have tempered the enthusiasm of both surgeons and institutions for widespread popularization of this technique. Therefore, in order to promote the application of flexible ureteroscopes, an inexpensive, small caliber, single-use flexible ureteroscope, with similar deflection capabilities as conventional integrated flexible ureteroscopes, may be a cost-effective option since it eliminates the associated repair costs and need for backup instruments.

The PolyScope ${ }^{\mathrm{TM}}$ (PolyDiagnost GmbH, Pfaffenhofen, Germany) is a disposable, modular design, flexible ureteroscope, which consists of a single-use flexible multilumen catheter and a separate, reusable optical fiber (Fig. 1). This system has been described in detail by Bader et al (6) and Johnson et al (7). Laser lithotripsy is an established endourological modality. Holmium lasers have broadened the applications for ureteroscopic stone treatment to include larger stone sizes throughout the entire upper urinary tract. The present study aimed to analyze the combined use of the PolyScope $^{\mathrm{TM}}$ and holmium laser lithotripsy for the treatment of renal and proximal ureteral calculi, in order to further evaluate the clinical value of this instrument. To the best of our knowl- 
edge, the present study represents the largest single-surgeon experience, in which there was uniformity of technique and treatment algorithms.

\section{Materials and methods}

Ethics statement. This study was approved by the ethics board of the First Hospital of Ningbo, Ningbo University (Ningbo, China). Written informed consent was not obtained, since all patient records were anonymized and de-identified prior to analysis.

Patients. A retrospective review of 691 procedures performed at the First Hospital of Ningbo, Ningbo University for renal and proximal ureteral calculi between June 2010 and June 2013 was conducted. The present study identified 382 consecutive patients with renal and proximal ureteral calculi who had undergone PolyScope ${ }^{\mathrm{TM}}$ and laser lithotripsy by a single surgeon (Dr Yue Cheng). A chart review was used to obtain patient, stone, and treatment parameters. The inclusion criteria for ureteroscopy in this series were: Renal and proximal ureteral calculi that failed extracorporeal shock wave lithotripsy (ESWL), obesity $\left(>30 \mathrm{~kg} / \mathrm{m}^{2}\right)$, poor candidates for percutaneous procedures, and patient preference. The exclusion criteria included severe hydronephrosis, stones in a calyceal diverticulum, and non-dependently draining collecting system (i.e., high insertion of the ureter, horseshoe kidney). All patients were offered ESWL and percutaneous nephrolithotomy (PNL) as alternative treatment options, and the final decision regarding the treatment was made by the patients themselves. Informed consent included specific mention of the possible need for numerous ureteroscopic procedures, residual stones and stent placement, and was obtained from all patients.

All patients underwent preoperative urine culture sensitivity tests, serum biochemistry and routine blood tests, a non-contrast spiral computed tomography (CT) urogram (to define total stone burden), and an intravenous urography. Patients with positive urine cultures were adequately treated with appropriate antibiotics, with all patients having a negative urine culture prior to surgery.

Surgical Procedures. All of the procedures were performed by a single surgeon (Fig. 2). Patients were administered intravenous preoperative antibiotics. Procedures for 153 patients were performed under spinal anesthesia, whereas 229 patients were operated on under general anesthesia. All procedures were carried out in the dorsal lithotomy position. A 22 F cystoscope was inserted into the bladder via the urethra, and the ureteral orifice was cannulated with an open-ended $5 \mathrm{~F}$ catheter and a 0.038 -inch hydrophilic guidewire. A $6 / 7.5 \mathrm{~F}$ or $8 / 9.8 \mathrm{~F}$ semi-rigid ureteroscope (Richard Wolf GmbH, Knittlingen, Germany) was passed over a guidewire using fluoroscopic guidance until it reached the proximal ureter or renal pelvis. This enabled dilation of the ureteral orifice and facilitated easier instrument passage during the flexible ureteroscopy. Subsequently, a ureteral access sheath (12/14 F or 14/16 F; Cook Medical, Inc., Bloomington, IN, USA) was placed in order to allow optimal visualization, maintain low intrarenal pressure, and to facilitate stone fragment extraction. If the ureteral access sheath could not be placed in patients with a tight ureter or ureteral stricture, a double-J stent was placed instead and the procedure was performed 2-6 weeks later. An 8 F PolyScope ${ }^{\mathrm{TM}}$ and a 200 or $365 \mu \mathrm{m}$ laser fiber were used for treatment. Irrigation was conducted using a $60 \mathrm{ml}$ syringe with normal saline connected to a 3-way stopcock with arterial line tubing. The holmium laser (Powersuite $100 \mathrm{~W}$; Lumenis, Inc., San Jose, CA, USA) was set to an energy level of $\mu$ 0.8-1.5 J and at a rate of $15-30 \mathrm{~Hz}$. Following lithotripsy, stone fragments were retrieved using a 1.9-Fr nitinol basket (Zero-Tip; Boston Scientific Japan Co. Ltd., Tokyo, Japan) if necessary. A double-J ureteral stent was routinely left in all patients, and the stent was removed 2-4 weeks postoperatively, when it was decided that the stent was no longer necessary.

A plain kidney, ureter, and bladder X-ray (KUB) was performed on postoperative day 1 in all cases, in order to assess the residual stone fragments and position of the double-J stent. A total of 1-2 weeks following the procedure, residual stone fragments were assessed again using a renal ultrasound, plain KUB film, and/or a CT scan. If necessary, a second-look procedure was scheduled 2-4 weeks following the first procedure. The operative time was calculated from the time of cystoscope insertion to the completion of stent placement. Stone-free status was defined as no fragments or asymptomatic, or the presence of clinically insignificant residual fragments $\leq 4 \mathrm{~mm}$, based on the European Association of Urology Guidelines 2013 (8).

Statistical analysis. Data are presented as the mean \pm standard deviation, the median and range for continuous variables, and the number and percentage for categorical variables. Data were recorded using Microsoft Excel 2010 software (Microsoft Corporation, Redmond, WA, USA).

\section{Results}

Table I presents patient demographics: 382 patients were eligible for the present study; 215 males and 167 females. The average age was 49.1 \pm 15.0 years (range, 17-84 years).

The stone demographics and operative data are shown in Table II. The mean stone size was $11.6 \pm 3.8 \mathrm{~mm}$ (range, 5-28 $\mathrm{mm}$ ), and the mean total stone burden was $16.7 \pm 5.7 \mathrm{~mm}$ (range, $8-46 \mathrm{~mm}$ ). A total of 305 patients $(79.8 \%$ ) had a stone burden $\leq 20 \mathrm{~mm}$, and 77 patients $(20.2 \%)$ had a stone burden $>20 \mathrm{~mm}$. Overall, 284 patients underwent one procedure, 87 patients underwent two procedures and 11 patients underwent three treatments, not including the final ureteroscopic inspection at the time of double-J stent removal. This equates to 491 primary procedures for 382 patients, a mean of $1.3 \pm 0.2$ primary procedures for each patient. The mean operative time per procedure was 67.1 $\pm 19.2 \mathrm{~min}$ (range, 35-116 min). Of all the 491 procedures, 121 multilumen catheters were used. The mean repeat usage of a multilumen catheter was 4.1 (range, 1-6). The stone-free rates following the first and the second procedures were 74.3 and $86.9 \%$, respectively. The overall stone-free rate was $86.9 \%$. Of the 11 patients who underwent a third procedure, none were rendered stone-free. The mean postoperative hospital stay following each procedure was $2.9 \pm 1.0$ days (range, $2-6$ days).

Table III presents stone clearance rates according to stone burden and location. The highest clearance rates were observed for proximal ureteral stones $(100 \%)$ and renal pelvic stones (88.7\%), whereas the lowest clearance rates were observed for 
Table I. Patient demographics.

\begin{tabular}{lc}
\hline Characteristics & Values \\
\hline Gender (n) & \\
Male & $215(56.3 \%)$ \\
Female & $167(43.7 \%)$ \\
Age $(\mathrm{y})$ & $49.1 \pm 15.0$ \\
BMI $\left(\mathrm{kg} / \mathrm{m}^{2}\right)$ & $23.6 \pm 2.2$ \\
Previous ESWL (n) & $274(71.7 \%)$ \\
\hline
\end{tabular}

Data from 382 patients. BMI, body mass index; ESWL, extracorporeal shock wave lithotripsy.

Table II. Stone demographics and operative data.

\begin{tabular}{lc}
\hline Variables & Values \\
\hline Mean stone size (mm) & $11.6 \pm 3.8$ \\
Mean stone burden (mm) & $16.7 \pm 5.7$ \\
Cumulative stone burden (n) & \\
$\leq 20$ mm & 305 \\
>20 mm & 77 \\
Stone location (n) & \\
Renal pelvis & 115 \\
Upper calyx & 96 \\
Middle calyx & 81 \\
Lower calyx & 43 \\
Proximal ureter & 29 \\
Multiple calyx & 18 \\
Mean number of procedures per patient & $1.3 \pm 0.2$ \\
Mean operative time per procedure (min) & $67.1 \pm 19.2$ \\
Mean repeat usage of the multilumen catheter & $4.1 \pm 1.5$ \\
Overall SFR (\%) & 86.9 \\
SFR after first treatment (\%) & 74.3 \\
SFR after second treatment (\%) & 86.9 \\
SFR after third treatment (\%) & 86.9 \\
Major complication rate (\%) & 2.1 \\
Minor complication rate (\%) & $6.9 \pm 1.0$ \\
Mean postoperative hospital stay (d) & \\
\hline
\end{tabular}

SFR, stone-free rate.

lower calyx stones (76.7\%) and multiple calyx stones $(77.8 \%)$ The higher the stone burden, the lower the postoperative stone-free rate ( $\leq 20$ vs. $>20.0 \mathrm{~mm} ; 89.8 \%$ vs. $75.3 \%$ ).

Ureteral perforation occurred in one patient, resulting in the discontinuation of the procedure following the placement of a double-J stent. This patient underwent a second treatment one month later (Clavien-Dindo grade III) (9). A follow-up CT urogram one month after double-J stent removal documented no stricture formation. Five patients exhibited significant
Table III. Stone-free rates for patients with various stone burdens and locations.

\begin{tabular}{lrr}
\hline Variables & Number & Overall SFR (\%) \\
\hline $\begin{array}{l}\text { Cumulative stone burden } \\
\leq 20 \mathrm{~mm}\end{array}$ & 305 & $274(89.8)$ \\
$>20 \mathrm{~mm}$ & 77 & $58(75.3)$ \\
Stone location & & \\
Renal pelvis & 115 & $102(88.7)$ \\
Upper calyx & 96 & $84(87.5)$ \\
Middle calyx & 81 & $70(86.4)$ \\
Lower calyx & 43 & $33(76.7)$ \\
Proximal ureter & 29 & $29(100)$ \\
Multiple calyx & 18 & $14(77.8)$ \\
\hline
\end{tabular}

SFR, stone-free rate.

bleeding, which resulted in poor visibility and led to discontinued procedures. However, no transfusions were necessary (Clavien-Dindo grade I). These five patients underwent an uncomplicated second treatment 2 weeks later, and no other major intraoperative complications (Clavien-Dindo grade III or greater), such as ureteral avulsion and renal rupture, were identified. Postoperative high-grade fever $>38.5^{\circ} \mathrm{C}$ was observed in 25 patients (Clavien-Dindo grade II), including two patients who developed sepsis (considered as a postoperative major complication). These patients were conservatively treated with parenteral administration of antibiotics.

\section{Discussion}

Rapid advances in distal-tip deflection, miniaturization of flexible ureteroscopes and corresponding accessories, have allowed the potential application of these instruments in not only diagnostic, but also therapeutic, procedures. However, the ongoing problems faced by surgeons with the conventional integrated flexible ureteroscope, including its low cost-effectiveness and fragility, ultimately limit the clinical application of these instruments $(10,11)$.

To decrease the overall cost of ureteroscopy by eliminating repair costs, a novel flexible ureteroscope $\left(\right.$ PolyScope $^{\mathrm{TM}}$ ) has been produced. The PolyScope ${ }^{\mathrm{TM}}$ is a flexible ureteroscope with a semi-disposable and modular design. The instrument has a separate optical system, which consists of a 10,000-pixel fiber optic ( $0.77 \mathrm{~mm}$ diameter) and an angle of view of $120^{\circ}$, therefore the image can be transmitted clearly and stably. The multilumen catheter of this instrument has an outer diameter of $8 \mathrm{~F}(2.65 \mathrm{~mm})$, a working/irrigation channel of $3.5 \mathrm{~F}(1.2 \mathrm{~mm})$, and a length of $85 \mathrm{~cm}$. The steering component is rotatable, which has a luer-lock mechanism for deflection of the steerable tip of the catheter. The tip deflection angle is $>240^{\circ}$, even when the $365 \mu \mathrm{m}$ laser fiber or the $3 \mathrm{~F}$ basket is inserted into the working channel. Bagley and Rittenberg (12) noted that a mean range of $140^{\circ}\left(104-175^{\circ}\right)$ of deflection is sufficient to reach the lower calyx. Therefore, theoretically, the PolyScope ${ }^{\text {тм }}$ is able to check all calyxes. The ocular, camera, and light cables are separated 


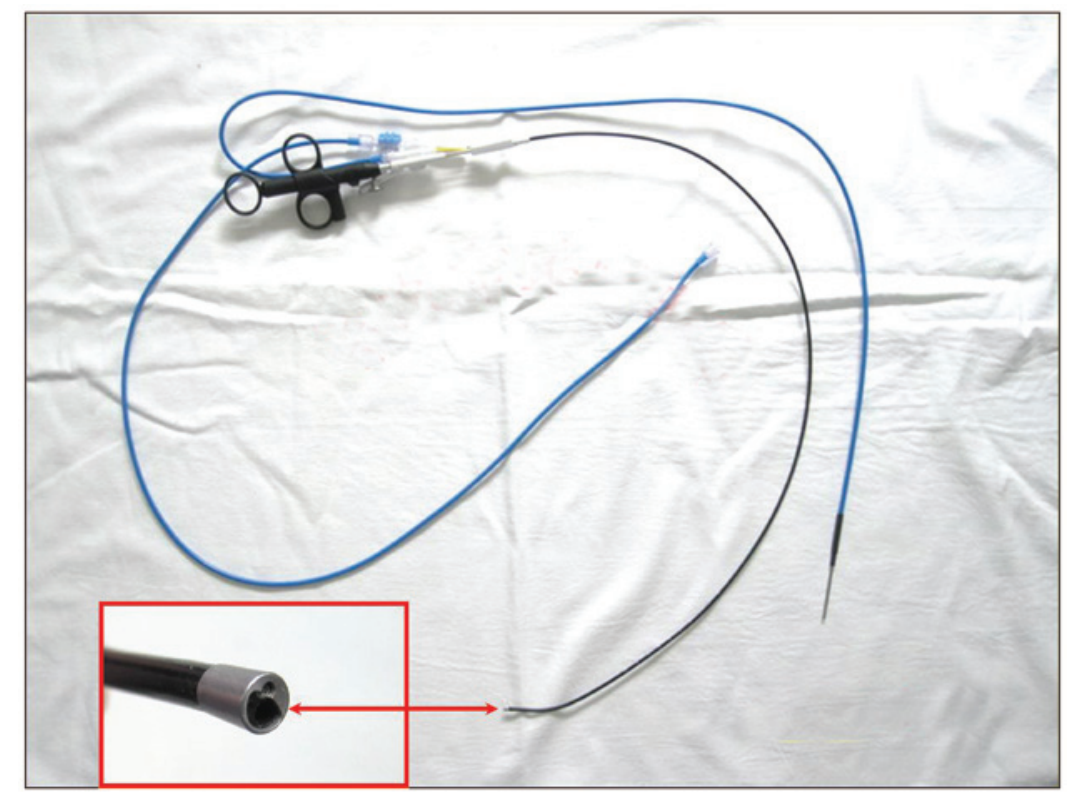

Figure 1. PolyScope $\mathrm{e}^{\mathrm{Tu}}$ ureteroscope consists of a single-use flexible multi-lumen catheter and a separate, reusable optical fiber.

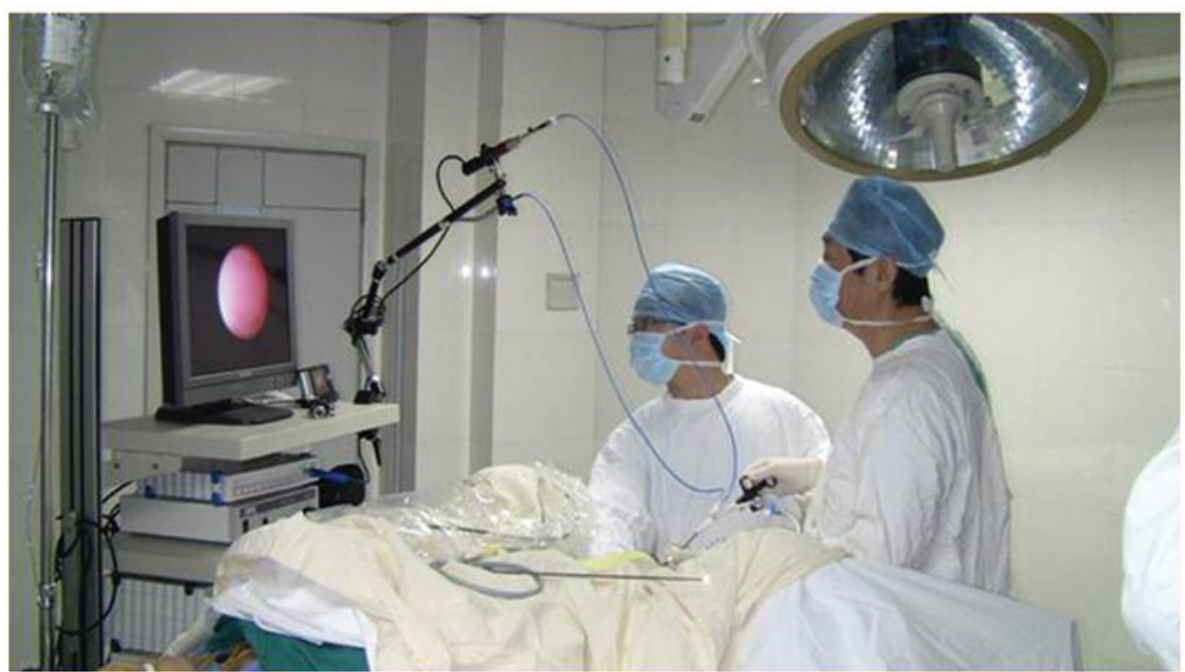

Figure 2. Dr Yue Cheng operating on a patient using PolyScope ${ }^{\circledR}$ and holmium laser lithotripsy.

from the endoscopic catheter outside the sterile field and are mounted on a four-joint arm, which allows for easy positioning of the non-sterile components relative to the operating side. As the separate image fiber does not come into direct contact with the patient, it does not require sterilization following each use. Therefore, the subsequent procedures can be performed immediately, simply by changing the multilumen catheter.

Bader et al (6) examined the clinical outcome of 32 patients with renal stones who were treated using the PolyScope ${ }^{\text {тм }}$, and reported a stone-free rate of $87.5 \%$. In addition, Bansal et al (13) diagnosed and treated 22 patients with the PolyScope ${ }^{\mathrm{TM}}$; of which six patients with undiagnosed hematuria were diagnosed with transitional-cell carcinoma, and 16 patients had stones $(<1 \mathrm{~cm})$ removed using a Dormia basket. Three PolyScope ${ }^{\mathrm{TM}}$ multilumen catheters were used to complete all of the procedures, indicating that the disposable catheters can be sterilized and reused. Gu et al (14) evaluated the clinical value of the PolyScope ${ }^{\mathrm{Tm}}$ endo- scope system in the treatment of 86 patients with upper urinary calculi with a diameter $<2 \mathrm{~cm}$. Lithotripsy was successful in 77 patients and the mean duration of the surgery was $42 \mathrm{~min}$.

In the present study, PolyScope ${ }^{\mathrm{TM}}$ and holmium laser lithotripsy were used to treat 382 patients with renal and proximal ureteral calculi; a total of 491 procedures were performed with 121 multilumen catheters, and the mean repeat usage of a multilumen catheter was 4.1. The stone-free rate following the first and the second procedures was 74.3 and $86.9 \%$, respectively. The third procedure did not improve the stone-free rate. The overall complication rate was $8.1 \%$. Six $(1.6 \%)$ intraoperative complications arose: One patient suffered from a ureteral perforation and five patients exhibited significant bleeding, which led to early discontinuation of the procedure. A total of 25 patients had postoperative high-grade fever $>38.5^{\circ} \mathrm{C}$. All of the patients fully recovered after appropriate treatment and no other severe complications were observed. The results of the present 
study are comparable with the conventional integrated flexible ureteroscope efficacy for treatment of upper urinary tract stones $(15,16)$. As for lower calyx calculi, theoretically there are no blind spots due to the excellent deflection of the instrument. However, due to anatomical particularities, the bending angle of the PolyScope ${ }^{\mathrm{TM}}$ was reduced following insertion of the auxiliary instruments; therefore some lower calyx calculi may not be reached. Therefore, the overall stone-free rate of lower calyx calculi was lower, as compared with that in the upper and middle calyx calculi. In addition, the overall stone-free rate was low in calculi with a cumulative stone burden $>20 \mathrm{~mm}$. Dasgupta et al (17) performed flexible ureterorenoscopy in 105 patients over a 9-month period, and obtained overall success in $72.3 \%$. A successful outcome was achieved in $72 \%$ with a stone size $\leq 10 \mathrm{~mm}, 80 \%$ for $11-20 \mathrm{~mm}$, and $50 \%$ for $>20 \mathrm{~mm}$. Two or more procedures were required in eight patients; thus suggesting that the flexible ureteroscope is more suitable for stones $<20 \mathrm{~mm}$. However, due to the minimally invasive nature of flexible ureteroscopy, staged procedure for the treatment of large stones is a practical option. In the present study, the stone-free rate was $74.3 \%$ after the first procedure, which increased to $86.9 \%$ after the second procedure. In a comparable study, Riley et al (18) used retrograde flexible ureteroscopy with holmium laser lithotripsy to treat 22 patients with stones $>2.5 \mathrm{~cm}$, the average number of procedures was 1.82, and overall stone-free rate was $90.9 \%$; thus suggesting that planned staged flexible ureteroscopy is a viable option for the treatment of renal stones $>2.5 \mathrm{~cm}$, with excellent stone-free results. These findings are consistent with the results of the present study.

According to the present study, the following points were particularly important for achieving higher stone-free results and minimizing complications: i) Prior to each flexible ureteroscopy, a semi-rigid ureteroscopy was performed to exclude ureteral stricture or other pathological findings. This enabled dilation of the ureteral orifice and facilitated enhanced instrument passage during the procedure; ii) following the semi-rigid ureteroscopy, a ureter access sheath was inserted at the level of the ureteropelvic junction to enable optimal visualization, allow numerous passages of the PolyScope ${ }^{\mathrm{TM}}$, maintain low intrarenal pressure and facilitate stone fragment extraction. In the case of a ureteral stricture or tight ureter, the ureteral access sheath could not be inserted; instead a double-J stent was placed for 2-6 weeks to passively dilate the ureter; iii) once the PolyScope ${ }^{\mathrm{TM}}$ was inserted into the pelvis, the operator identified the ureteropelvic junction as a mark, gradually distinguished the upper calyx, middle calyx and lower calyx, and then searched for stones calyx by calyx; iv) in general, the holmium laser lithotripsy was set at a maximum power of 20-45 $\mathrm{W}(0.8-1.5 \mathrm{~J} / 15-30 \mathrm{~Hz})$. The laser energy and frequency of pulsation varied according to different stone densities and stone burdens. In order to convert the stone center to small fragments and fine dust, higher settings were employed initially; however, the settings were decreased to minimize kinetic forces and emphasize mechanical fragmentation once the residual mass became mobile.

There are some limitations to the present study. The present study is a retrospective review from a single institution. In addition, a single-surgeon experience obviously has limitations with regards to the reproducibility of the results.

In the present study, the modular flexible ureteroscope $\left(\right.$ PolyScope $^{\mathrm{TM}}$ ) proved its safety, utility and efficacy, and we suggest that modular flexible ureteroscopy with holmium laser lithotripsy may be considered the primary method for the diagnosis and treatment of renal and proximal ureteral calculi in select patients. Notably, the modular design of the PolyScope ${ }^{\mathrm{TM}}$ avoids high maintenance costs associated with the conventional integrated flexible ureteroscope, making the PolyScope ${ }^{\mathrm{TM}}$ a superior and more cost-effective option for clinical use. Nevertheless, further clinical studies over a longer period of time, with a larger patient cohort and at different centers, are required to validate the perceived advantages of this device.

\section{Acknowledgements}

A research grant was provided by the Ningbo Social Development Projects Foundation (No. 2011C50065).

\section{References}

1. Marshall VF: Fiber optics in urology. J Urol 91: 110-114, 1964.

2. Afane JS, Olweny EO, Bercowsky E, Sundaram CP, Dunn MD, Shalhav AL, McDougall EM and Clayman RV: Flexible ureteroscopes: A single center evaluation of the durability and function of the new endoscopes smaller than 9Fr. J Urol 164: 1164-1168, 2000.

3. Landman J, Lee DI, Lee C and Monga M: Evaluation of overall costs of currently available small flexible ureteroscopes. Urology 62: 218-222, 2003.

4. Carey RI, Gomez CS, Maurici G, Lynne CM, Leveillee RJ and Bird VG: Frequency of ureteroscope damage seen at a tertiary care center. J Urol 176: 607-610, 2006.

5. Canales BK, Gleason JM, Hicks N and Monga M: Independent analysis of Olympus flexible ureteroscope repairs. Urology 70: 11-15, 2007.

6. Bader MJ, Gratzke C, Walther S, Schlenker B, Tilki D, Hocaoglu Y, Sroka R, Stief CG and Reich O: The PolyScope: A modular design, semidisposable flexible ureterorenoscope system. J Endourol 24: 1061-1066, 2010.

7. Johnson MT, Khemees TA and Knudsen BE: Resilience of disposable endoscope optical fiber properties after repeat sterilization. J Endourol 27: 71-74, 2013.

8. Tiselius HG, Ackermann D, Allen P, Buck C, Conort P and Gallucci M; Working Party on Lithiasis, European Association of Urology: Guidelines on urolithiasis. Eur Urol 40: 362-371, 2001.

9. Mitropoulos D, Artibani W, Graefen M, Remzi M, Rouprêt M and Truss M; European Association of Urology Guidelines Panel: Reporting and grading of complications after urologic surgical procedures: An ad hoc EAU guidelines panel assessment and recommendations. Eur Urol 61: 341-349, 2012.

10. Defidio L, De Dominicis M, Di Gianfrancesco L, Fuchs G and Patel A: Improving flexible ureterorenoscope durability up to 100 procedures. J Endourol 26: 1329-1334, 2012.

11. Sooriakumaan P, Kaba R, Andrews HO and Buchholz NP: Evaluation of the mechanisms of damage to flexible ureteroscopes and suggestions for ureterorenoscopy preservation. Asian J Androl 7: 433-438, 2005.

12. Bagley DH and Rittenberg MH: Percutaneous antegrade flexible ureteroscopy. Urology 27: 331-334, 1986.

13. Bansal H, Swain S, Sharma GK, Mathanya M, Trivedi S, Dwivedi US and Singh PB: Polyscope: A new era in flexible ureterorenoscopy. J Endourol 25: 317-321, 2011.

14. Gu SP, Huang YT, You ZY,Zhou X, Lu YJ, He CH and Qi J: Clinical effectiveness of the PolyScope ${ }^{\mathrm{TM}}$ endoscope system combined with holmium laser lithotripsy in the treatment of upper urinary calculi with a diameter of less than $2 \mathrm{~cm}$. Exp Ther Med 6: 591-595, 2013.

15. Breda A, Ogunyemi O, Leppert JT and Schulam PG: Flexible ureteroscopy and laser lithotripsy for multiple unilateral intrarenal stones. Eur Urol 55: 1190-1196, 2009.

16. Cohen J, Cohen S and Grasso M: Ureteropyeloscopic treatment of large, complex intrarenal and proximal ureteral calculi. BJU Int 111: E127-E131, 2013.

17. Dasgupta P, Cynk MS, Bultitude MF, Tiptaft RC and Glass JM: Flexible ureterorenoscopy: Prospective analysis of the Guy's experience. Ann R Coll Surg Engl 86: 367-370, 2004.

18. Riley JM, Stearman L and Troxel S: Retrograde ureteroscopy for renal stones larger than $2.5 \mathrm{~cm}$. J Endourol 23: 1395-1398, 2009. 